\title{
High Sensitivity Refractive Index Sensor based on Large-angle Tilted Fiber Grating with Carbon Nanotube Deposition
}

\author{
Abdulyezir A. Badmos ${ }^{a}$, Qizhen Sun ${ }^{a}$, Zhijun Yan ${ }^{a}$, Raz N Arif ${ }^{a}$, Junxi Zhang $^{a}$, \\ Alex Rozhin ${ }^{a}$, Lin Zhang $^{a, *}$ \\ ${ }^{a}$ Aston University, Aston Institute of Photonic Technologies, Engineering and Applied Science, \\ Aston triangle, Birmingham, UK, B4 7ET
}

\begin{abstract}
This paper presents a highly sensitive ambient refractive index (RI) sensor based on $81^{\circ}$ tilted fiber grating $\left(81^{\circ}\right.$-TFG) structure UV-inscribed in standard telecom fiber $(62.5 \mu \mathrm{m}$ cladding radius) with carbon nanotube (CNT) overlay deposition. The sensing mechanism is based on the ability of CNT to induce change in transmitted optical power and the high sensitivity of $81^{\circ}$-TFG to ambient refractive index. The thin CNT film with high refractive index enhances the cladding modes of the TFG, resulting in the significant interaction between the propagating light and the surrounding medium. Consequently, the surrounding RI change will induce not only the resonant wavelength shift but also the power intensity change of the attenuation band in the transmission spectrum. Result shows that the change in transmitted optical power produces a corresponding linear reduction in intensity with increment in RI values. The sample shows high sensitivities of $\sim 207.38 \mathrm{~nm} / \mathrm{RIU}, \sim 241.79 \mathrm{~nm} / \mathrm{RIU}$ at RI range $1.344-1.374$ and $\sim 113.09 \mathrm{~nm} / \mathrm{RIU}, \sim 144.40 \mathrm{~nm} / \mathrm{RIU}$ at RI range 1.374-1.392 (for X-pol and Y-pol respectively). It also shows power intensity sensitivity of $\sim 65.728 \mathrm{dBm} / \mathrm{RIU}$ and $\sim 45.898$ (for X-pol and Y-pol respectively). The low thermal sensitivity property of the $81^{\circ}$-TFG offers reduction in thermal cross-sensitivity and enhances specificity of the sensor.
\end{abstract}

Keywords: Tilted Fibre grating, carbon nanotubes, ambient medium, refractive index, cross-sensitivity, attenuation band, optical power, resonant wavelength, wavelength shift, sensitivity,

\section{INTRODUCTION}

Optical fibre gratings have been relentlessly used for sensing applications over the last few decades as a result of their real time monitoring capability ${ }^{1-4}$, high sensitivity and selectivity to external perturbation such as temperature, pressure, bending, humidity, strain and refractive index variations ${ }^{5-10}$. Refractive index (RI) sensors are based on the principle that variations in RI of ambient medium affect the resonant peaks of mode coupling induced by the optical fibre grating. This is only achievable by optical gratings that enable coupling between the core and the cladding modes with evanescence field diffusing into the surrounding medium ${ }^{11,12}$. Detection of variation in RI is important in application such as water treatment plants for purity level indication. Research findings reported highly sensitive RI sensors combining LPGs and tapered fibres ${ }^{13}$, microfiber-based Mach-Zehnder interferometer ${ }^{14}$, multimode interference ${ }^{3}$, SPRbased optical fibre sensors using gold-silver alloy particles ${ }^{1}$, optical microfiber and fibre tapering ${ }^{15-18}$; while these RI sensors have achieved high sensitivity they are mainly based on wavelength demodulation.

Large angle tilted fibre grating (also often termed excessively tilted fibre grating (Ex-TFG)) is one of the optical gratings that couple the fundamental core mode to the forward propagating cladding modes; the other being Long period grating (LPG). Theoretical modelling and analysis reveals higher sensitivity ( 1000 times) exhibited by forward-propagating cladding modes in comparison with the backward-propagating ones ${ }^{19}$. Ex-TFGs, first reported by ${ }^{20}$, due to the asymmetric structure induced by their excessively tilted fringes have dual-peak resonances in their transmission spectrum corresponding to two sets of orthogonal polarisation dependent modes ${ }^{20,21}$. They demonstrate much higher

Optical Sensing and Detection IV, edited by Francis Berghmans, Anna G. Mignani, Proc. of SPIE Vol. 9899, $989916 \cdot$ C 2016 SPIE · CCC code: 0277-786X/16/\$18 · doi: 10.1117/12.2227703 
sensitivity in lower RI region compared to normal LPG since they are capable of coupling light to much higher order cladding modes.

In this paper, we propose a novel high sensitive RI sensor based on $81^{\circ}$ - tilted fibre grating UV-inscribed in standard communication fibre (SMF-28) with carbon nanotube (CNT) overlay deposition on the grating area. Besides having high RI and high absorption of light ${ }^{22}$, CNT are highly compatible to silica surface forming thin film overlay ${ }^{23,24}$. As obtainable in previous RI sensors where sensing principles are based only on wavelength sensitivity, this sensor combines the demodulation of both wavelength and intensity variations of the resonant dip.

\section{FABRICATION}

The fabrication process of this sensor includes two steps: (1) Large-angle TFG fabrication, and (2) CNT deposition on grating area of fibre. Firstly, the Ex-TFG was fabricated by a $244 \mathrm{~nm}$ UV source from frequency doubled Argon laser (Coherent Sabre Fred) using a custom-designed amplitude mask of period $6.6 \mu \mathrm{m}$. The period of the amplitude mask was specially designed to ensure generated grating response from higher order cladding modes are centred in the C-L band of the frequency windows. Standard communication fibre (SMF-28) hydrogen loaded under high temperature $\left(80^{\circ} \mathrm{C}\right)$ and high pressure (150 bars) for 48 hours to enhance UV- photosensitivity was employed for the fabrication. During the inscription process the amplitude mask was tilted at $\sim 77^{\circ}$ external angle in other to achieve excessively tilted fringes at $\sim$ $81^{\circ}$ in the fibre core. The relationship between the external tilted, internal tilted angle and the grating period is expressed as ${ }^{25}$ :

$$
\begin{gathered}
\Lambda_{G}=\frac{\Lambda_{A M} \cos \left(\frac{\pi}{2}-\tan ^{-1}\left[\frac{1}{n_{U V} \tan \left(\theta_{e x t}\right)}\right]\right)}{\cos \theta_{e x t}} . \\
\theta_{\text {int }}=\frac{\pi}{2}-\tan ^{-1}\left[\frac{1}{n_{U V} \tan \left(\theta_{e x t}\right)}\right] .
\end{gathered}
$$

Where $\Lambda_{G}$ and $\Lambda_{A M}$ are the normal grating period inside the fibre and the amplitude mask respectively, $\theta_{\text {int }}$ is the internal angle inside the fibre, $\theta_{e x t}$ is the external tilted angle of the mask and $n_{U V}$ is the refractive index of the fibre at UV-source wavelength (244nm).

Secondly, the CNT deposition was overlaid by a relatively simple and effective method on the grating area of the $81^{\circ}$ TFG for easy repeatability of the sensor. The CNT solution was prepared by dispersing $0.5 \mathrm{mg}$ of CNT powder in $10 \mathrm{ml}$ of N-methyl-2-pyrrolidone (NMP) solution and sonicated in water bath $(20 \mathrm{kHz}, 200 \mathrm{~W}, 1 \mathrm{~h}$, Nanoruptor, Diagenode SA, Liege, Belgium). NMP was used because of its efficiency in in the direct dispersion of hydrophobic materials (CNT) at lower concentrations $(<0.02 \mathrm{mg} / \mathrm{ml}){ }^{26}$. Polyvinyl pyrrolidone (PVP) polymer $(1 \mathrm{mg} / \mathrm{ml})$ was added as dispersion agent in order to enhance the concentration of CNTs within the resulting dispersion ${ }^{27}$. Thereafter, the CNT-PVP-NMP solution was centrifuged for 30 minutes at 10000 RMP with MLS-50 rotor (Optima MaxXP Benchtop Ultracentrifuge, Beckman Coulter, Brea, California, USA) with a view to removing residual CNT bundles and achieving a highly uniform dispersion. The well-dispersed CNT solution was deposited on the $81^{\circ}$-TFG by placing the TFG in a microcapillary tube filled with the CNT dispersion for a couple of minutes and repeated several times. The entire surface of the grating region (of grating length $24 \mathrm{~mm}$ ) was totally submerged in the CNT solution and allowed to dry in air at atmospheric pressure for 24 hours. This method is found to be cost effective as it only entails the mechanical transfer of cold nanomaterials with no heating required ${ }^{23}$. CNTs being cylindrical carbon molecules structured with diameter of few nanometres and high surface area for absorption of water molecules and aqueous solutions, have been reported to have their refractive index (the effective dielectric property of CNT) vary with changes in RI of ambient medium ${ }^{28}$. 


\section{WORKING PRINCIPLE}

The working principle includes two aspects: wavelength shift and intensity variation. The large-angle TFG induces two different sets of birefringence cladding modes (TM/TE resonance peaks) coupling and the resulting strongest coupling between the fundamental core mode and the co-propagating cladding modes is expressed by the phase matching condition ${ }^{21}$ :

$$
\begin{gathered}
\lambda_{m, r e s}=\left(n_{c o}^{e f f}\left(\lambda_{m, r e s}, n_{1}, n_{2}\right)-n_{c l, m}^{i, e f f}\left(\lambda_{m, r e s}, n_{2}, n_{S R I}\right)\right) \frac{\Lambda_{G}}{\cos \theta} \quad i=T E \text { or } T M \\
\text { the axial period is expressed as: } \quad \Lambda=\frac{\Lambda_{G}}{\cos \theta} .
\end{gathered}
$$

where $\lambda_{m, r e s}$ is the resonance wavelength due to coupling between fundamental core mode and the $m^{\text {th }}$ cladding mode, $n_{c o}^{e f f}$ is the effective index of the fundamental core mode, $n_{c l, m}^{i, e f f}$ is the effective index of $m^{\text {th }}$ cladding mode at the resonance wavelength, $n_{S R I}$ is the refractive index of ambient medium, $n_{1}, n_{2}$ are the RI of the core and cladding respectively.

For intensity variation based sensing, the principle is as follows: when the surrounding RI changes, it leads to a variation in the amount of attenuation of the cladding modes which would result in change in the attenuation depth of the attenuation band. Therefore, the sensing of the RI of the external surrounding medium can be obtained by monitoring the amount of attenuation in the attenuation bands of the TFG. And for wavelength shift based sensing; as the RI of surrounding medium varies there exists a corresponding variation in the effective index of the cladding modes, and then the resonant wavelength will shift according to equation (3). Especially when the RI of the ambient medium is less than that of the cladding, then with every increase in RI of the ambient medium the effective RI of the cladding also increases while that of the fundamental core remains constant resulting in red shift of the resonance wavelength.

Meanwhile, with additional thin film (CNT layer) on the cladding of the $81^{\circ}$-TFG, the sensor is now treated as an ExTFG surrounded by a medium of infinite thickness. It can be modified to incorporate additional dielectric layer between cladding of finite thickness and the surrounding medium of infinite thickness. The schematic diagram in fig. 1 shows the sensor as a model of four-layer cylindrical waveguide. The TFG, now surrounded by medium of higher RI, will have a portion of the power of cladding mode reflected by the CNT layer while the remaining are transmitted ${ }^{29}$. Quantification of the amount of reflectance of the cladding mode at the fibre cladding/ CNT layer interface can be obtained as follows ${ }^{30}$ :

$$
\begin{gathered}
R=\left|\frac{r_{23}+r_{34} e^{-i \tilde{k}_{c n t}}}{1+r_{23} r_{34} e^{-i \tilde{k}_{c n t}}}\right|^{2} . \\
r_{23}=\frac{n_{\text {clad }}-\tilde{n}_{c n t}}{n_{c l a d}+\tilde{n}_{c n t}} .
\end{gathered}
$$




$$
\begin{gathered}
r_{34}=\frac{\tilde{n}_{c n t}-n_{S R}}{\tilde{n}_{c n t}+n_{S R}} . \\
\tilde{k}_{c n t}=\frac{4 \pi \tilde{n}_{c n t} d_{c n t}}{\lambda}=\frac{4 \pi n d_{c n t}}{\lambda}-i \frac{4 \pi k d_{c n t}}{\lambda}=\beta_{c n t}-i a d_{c n t} .
\end{gathered}
$$

where $a=4 \pi k / \lambda$ is the absorption coefficient of the thin CNT layer, $n_{\text {clad }}$ and $n_{S R}$ are the RIs of the cladding and the surrounding medium respectively, $\tilde{n}_{c n t}=n-i k, r_{i, j}$ in equations (6) and (7) is the reflection coefficient of the interface between each two layers. Figure 1 shows each distinct layer as components of the sensor and from equations (5), (6), (7) and (8) the reflectance, R would vary with $n_{S R}$ which consequently leads to variation in the amount of attenuation of the cladding modes resulting in change in attenuation depth of the attenuation band.

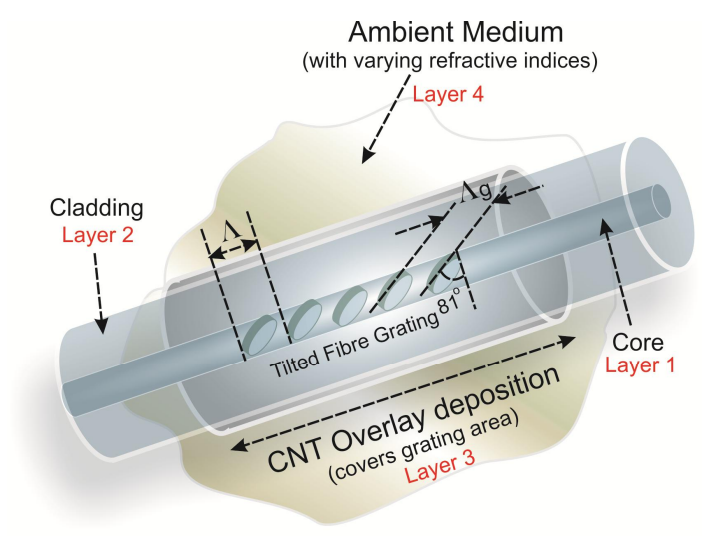

Figure 1: Schematic of the CNT-coated $81^{\circ}-\mathrm{TFG}$ RI sensor

Precisely, this sensor is based on the principle that both the intensity and the wavelength of the CNT-coated $81^{\circ}-\mathrm{TFG}$ changes with changes in the RI of ambient medium. The CNT overlay deposition is responsible for the power modulation of the sensor while the $81^{\circ}-\mathrm{TFG}$, being highly sensitive to ambient RI variations, induces the wavelength shift as each solution is administered.

\section{EXPERIMENTAL SETUP}

The experimental setup of the CNT coated TFG based RI sensor, as shown in figure 2, includes a broadband light source (Fianium ultrafast fibre laser- super continuum- range $850 \mathrm{~nm}$ to far infrared), an optical spectrum analyser (OSA), micrometre stage, an in-fibre polarizer and a polarization controller. Light from the super-continuum laser was passed through a polarizer (broadband internal polarizer for polarimeter PAT 9000B) and a polarisation controller (manual fibre paddle polarization controller) and transmitted through the sample (CNT coated TFG) with the transmission spectrum displayed on the optical spectrum analyser (Model 86140 Agilent) with resolution of $1.0 \mathrm{~nm}$ and average power of -33.2 $\mathrm{dBm}$. The polarization controller makes switching form the TE to TM modes of the cladding modes possible and each mode is subjected to variation in the surrounding RI. We evaluated the transmission spectra of $81^{\circ}$-TFG ranging from 1250 to $1650 \mathrm{~nm}$. As peculiar with Ex-TFGs, the peaks split into two which is as a result of the coupling of the two sets of cladding modes of orthogonal polarization when measured using unpolarised light and polarized light with orthogonal 
polarizations ${ }^{20}$. A pair of the dual-peak spectrum centred around $1550-1580 \mathrm{~nm}$ was zoomed for the refractive index sensing as shown in figure 3 below.

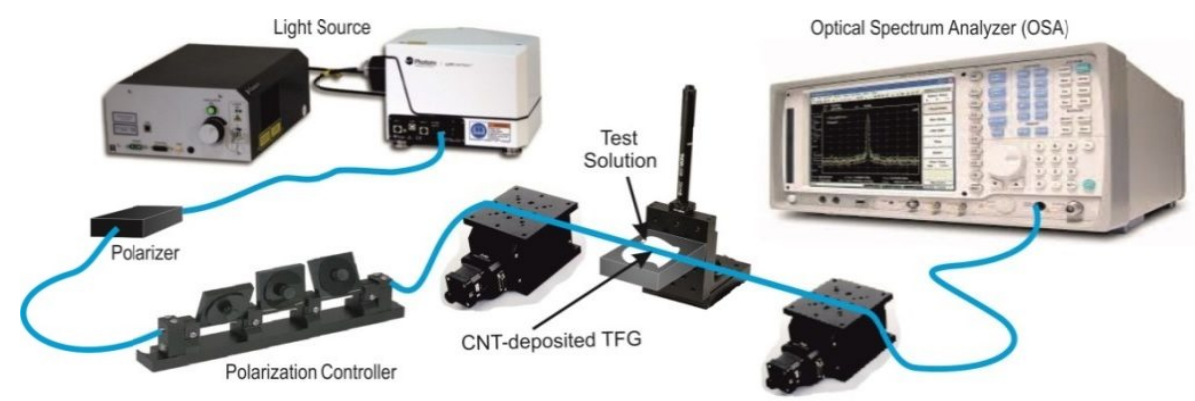

Figure 2: Experimental setup of RI sensing of the CNT-coated $81^{\circ}$-TFG RI sensor

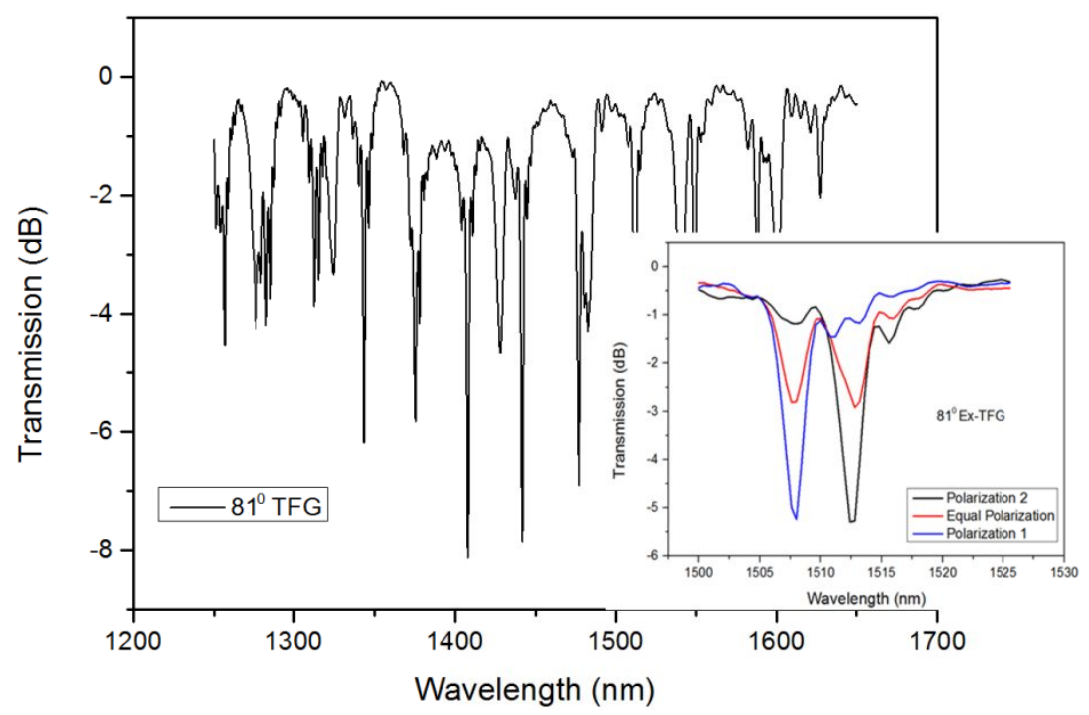

Figure 3: Transmission spectra of $81^{\circ}$-TFG showing series of dual-peak resonances from 1250 to $1650 \mathrm{~nm}$; (inset: zoomed dual peak at around $1500 \mathrm{~nm}$ when launched with randomly polarized light and orthogonally polarized light).

The test solution used for this experiment was prepared from the dissolution of $+\mathrm{D}+$ glucose (purchased from Sigma Aldrich) in de-ionized water and stirred under room temperature of about $22 \pm 0.1^{\circ} \mathrm{C}$ until a saturated glucose solution was formed. Different concentration of the glucose solution was obtained by varying the percentage concentration of the glucose powder and the volume of the de-ionized water. The refractive index of each solution was obtained using a digital refractometer with resolution of 0.001 . To demonstrate the effect of the CNT film on the TFG, we compare the RI sensing performance between bare TFG and the CNT coated TFG. The RI solution ranges from 1.300 to 1.370 for the experiment with the bare fibre while that for the CNT deposited fibre ranges from $1.344-1.392$. This was because the experiment was carried out at different points in time and new glucose solutions have to be made. And all the measurements were carried out indoor at room temperature. Since Ex-TFGs are less sensitive to temperature compared to LPGs, the fear of temperature-induced perturbation does not come to play. 
During the experiment, the CNT-coated $81^{\circ}-\mathrm{TFG}$ was mounted on the micrometre stage and glass slides were placed repeatedly directly under the grating area as each solution is examined for RI sensing. At these times, the CNT coated grating region was totally submerged in the glucose solution with a view to getting accurate results and the transmission response was monitored on the OSA. After each measurement, the CNT-coated $81^{\circ}$-TFG was rinsed with methanol and the spectrum monitored to initial position in air. Both the in-fibre polarizer and polarization controller enables switching from one orthogonal state to the other (TE and TM cladding modes).

\section{RESULTS AND DISCUSSION}

With the CNT overlay on the grating region of the $81^{\circ}-\mathrm{TFG}$, the sensor, as shown in figure 4 , clearly display distinct power levels as well as resonant wavelength shifts with test solutions of different RI. It is observed that as the ambient RI varies lower reflectance occurs at higher RI values and higher reflectance at lower RI values. In other words, as the RI of the ambient solution increases, the intensity minima of the attenuation bands of the orthogonal TE and TM cladding mode decreases. This shows that with the CNT overlay on the fibre, the cladding modes extend well into the radiation mode. Then the amount of reflectance at the cladding/CNT boundary would depend on the surrounding medium RI as described in equation (5). Also, the wavelength notably shifts towards the longer wavelengths (red-shift) as RI of surrounding medium varies.

Figure 5(a) shows the intensity plot of TE/TM cladding modes against RI of ambient solution and it is obvious that there exists positive correlation between them. A sensitivity of $\sim 68.728 \mathrm{~dB} / \mathrm{RIU}$ and $\sim 45.898 \mathrm{~dB} / \mathrm{RIU}$ were recorded for X-pol and Y-pol respectively. Also, as shown in figure 5(b), the wavelength shift for both TE and TM modes of the TFG are plotted. As the surrounding RI varies, the wavelength is observed to shift towards longer wavelengths spanning slightly over $15 \mathrm{~nm}$ for the entire measurement. The sensor achieved high sensitivity of $\sim 207.38 \mathrm{~nm} / \mathrm{RIU}, \sim 241.79 \mathrm{~nm} / \mathrm{RIU}$ at RI range 1.344-1.374 and $\sim 113.09 \mathrm{~nm} / \mathrm{RIU}, \sim 144.40 \mathrm{~nm} / \mathrm{RIU}$ at RI range 1.374-1.392 (for X-pol and Y-pol respectively).
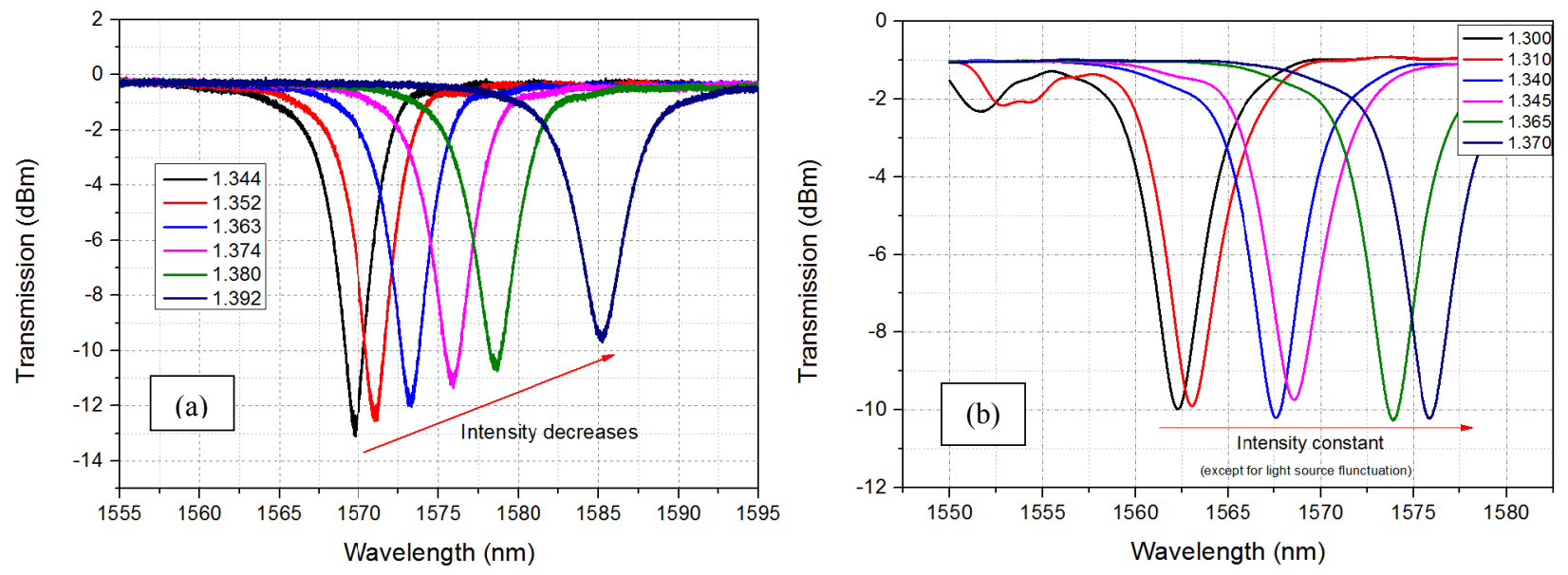

Figure 4: Transmission spectrum of the ambient RI sensing of (a) CNT-coated $81^{\circ}$-TFG (b) bare $81^{\circ}$-TFG

Meanwhile, we test the sensing performance of the bare TFG for comparison. The transmission spectrum of $81^{\circ}-\mathrm{TFG}$ (bare fibre) in figure 4(b) demonstrates that only resonant wavelength is sensitive to the SRI variation, while the intensity of the resonant dip varies randomly but almost constant when the grating region was submerged in different RI ambient solutions. So the bare TFG based RI sensor can only be achieved by measuring the wavelength shift. 

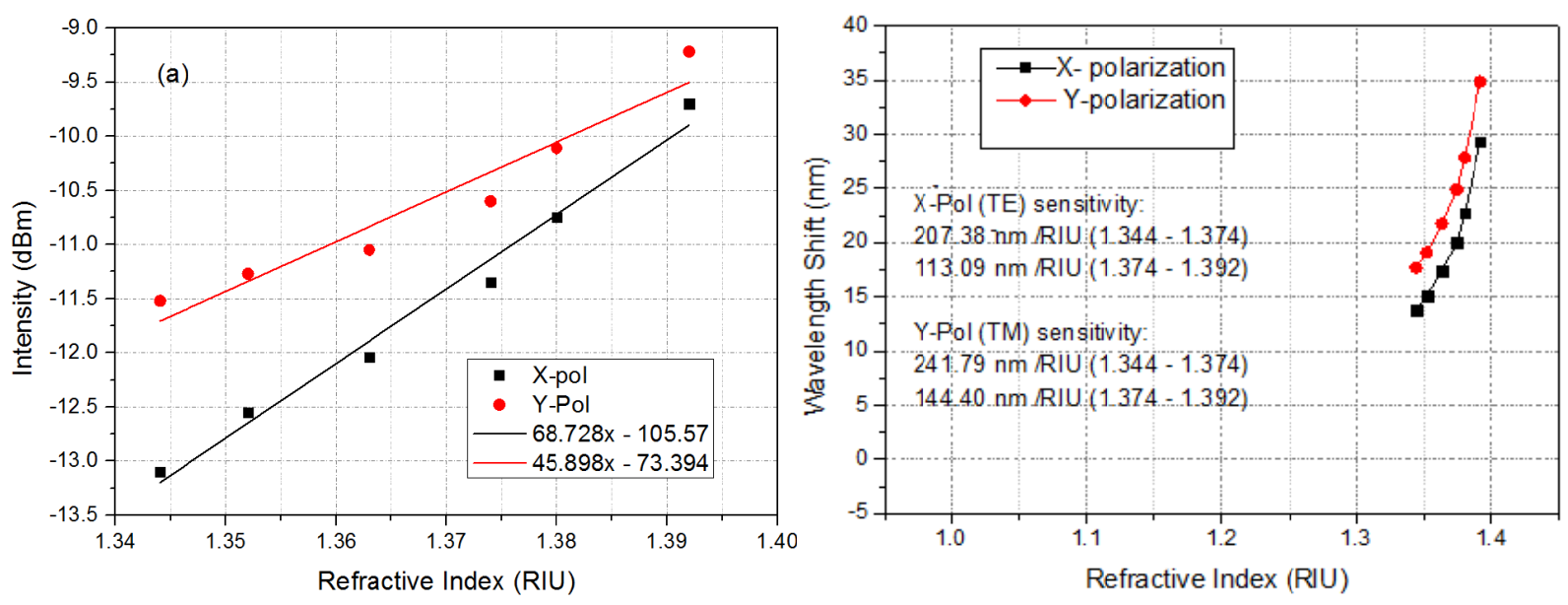

Figure 5: (a) Plot of intensity variation of the TE/TM cladding modes (X-pol, Y-pol) against RI of ambient solution (b) Wavelength shift curve

\section{CONCLUSION}

In summary, we have demonstrated a novel highly sensitive ambient refractive index (RI) sensor based on $81^{\circ}$ tilted fibre grating $\left(81^{\circ}-\mathrm{TFG}\right)$ structure UV-inscribed in standard telecom fibre $(62.5 \mu \mathrm{m}$ cladding radius) with carbon nanotube (CNT) overlay deposition. The surrounding RI change will induce not only the resonant wavelength shift but also the power intensity change of the attenuation band in the transmission spectrum. For the wavelength demodulation, the sensor achieved high sensitivity of $\sim 207.38 \mathrm{~nm} / \mathrm{RIU}, \sim 241.79 \mathrm{~nm} / \mathrm{RIU}$ at RI range $1.344-1.374$ and $\sim 113.09 \mathrm{~nm} / \mathrm{RIU}$, $\sim 144.40 \mathrm{~nm} / \mathrm{RIU}$ at RI range 1.374-1.392 (for X-pol and Y-pol respectively); while for the intensity demodulation, it recorded a sensitivity of $\sim 68.728 \mathrm{~dB} / \mathrm{RIU}$ and $\sim 45.898 \mathrm{~dB} / \mathrm{RIU}$ for X-pol and Y-pol respectively. This sensor finds practical applications in bio/chemical detection due to its high RI sensitivity, low temperature cross-sensitivity, low cost power-based demodulation and strong mechanical strength. 


\section{REFERENCES}

1 Tu, H., Sun, T., and Grattan, K. T. "SPR-based optical fiber sensors using gold-silver alloy particles as the active sensing material," IEEE Sensors Journal 13, 2192-2199 ((June (6)) (2013)).

2 Mitsushio, M., Higashi, S., and Higo, M. "Construction and evaluation of a gold-deposited opticalfiber sensor system for measurements of refractive indices of alcohols," Sens. Actuators A 111, 252-259 ((March(2-3)) (2004)).

3 Silva, S., Frazao, O., Santos, J. L., Malcata, F. X. "A reflective optical fibre refractometer based on multimode interference," Sens. Actuator B 161, 88-92 ((January (1)) (2012)).

4 Consolates, M., Crescitelli, A., Penza, M., Aversa, P., Veneri, P. D., Giordano, M., Cusano, A. " SWCNT nano-composite optical sensors for VOC and gas trace detection," Sens. Actuators B 138, 351-361 ((April (1)) (2009)).

5 Shivananju, B. N., Yamdagni, S., Fazuldeen, R., Kumar, A. K., Nithin, S. P., Varma, M. M., and Asokan, S. " Highly Sensitive Carbon Nanotubes Coated Etched Fiber Bragg Grating Sensor for Humidity Sensing," IEEE Sensors Journal 14, 2615-2619 (August 2014).

6 Li, X., Wang, D., Zhao, F., and Dai, E. " Simultaneous independent temperature and strain measurement using one fiber Bragg grating based on the etching technique," Microwave and Optical Technology Letters 43, 478$481((2004))$.

$7 \quad$ King, D., Lyons, W.B., Flanagan, C., Lewis, E. " A multipoint optical fibre sensor system for use in process water systems based on artificial neural network pattern recognition techniques," Sens. Actuators A (115), 293302 (September (2004)).

8 Ji, W.B., Liu, H. H., Tjin, S.C., Chow, K.K., Lim, A. "Ultrahigh sensitivity refractive indexsensor based on optical microfiber," IEEE Photon. Technol. Lett 24 1872-1874 ((October (20))(2012) ).

9 Kim, K. S., Mizuno, Y., Nakano, M., Onoda, S., Nakamura, K. " Refractive index sensor for liquids and solids using dielectric multilayer films deposited on optical fiber end surface," IEEE Photon. Technol. Lett. 23 14721474 ((October (20)) (2011)).

10 Wang, P.F., Brambilla, G., Ding, M., Semenova, Y., Wu, Q., Farrell, G. " High-sensitivity, evanescent field refractometric sensor based on a tapered multimode fiber interference," Opt. Lett. 36, 2233-2235 ((June (12)) (2011)).

11 Fan, X., White, I. M., Shopova, S. I., Zhu, H., Suter, J. D. and Sun, Y. "Sensitive optical biosensors for unlabeled targets: A review," Anal. Chem. Acta 620, p. 19 (2008).

12 Baldini, F. B. M., Chiavaioli, F., Giannetti, A., and Trono, C. "Optical fibre gratings as tools for chemical and biochemical sensing," Anal. Bioanalytical Chemistry 402, p. 8 (2012).

13 Allsop, T., Floreani, F., Jedrzejewski, K.P., Marques, P.V.S., Romero, R., Webb, D.J., Bennion, I. " Spectral characteristics of tapered LPG device as a sensing element for refractive index and temperature," J. Light. Technol. 24, 870-878. (2006).

14 Wo, J. H., Wang, G.H., Cui, Y., Sun, Q.Z., Liang, R.B., Shum, P.P., Liu, D.M. " Refractive index sensor using microfiber-based Mazh-Zehnder interferometer," Opt. Lett. 37, 67-69 (2012).

15 Kieu, K. Q., Mansuripur, M. " Biconical fiber taper sensors," IEEE Photon. Technol.Lett., 2239-2241 (November (24) (2006)).

16 Lu, P., Men, L.Q., Sooley, K., Chen, Q.Y. "Tapered fiber Mach-Zehnder interferome-ter for simultaneous measurement of refractive index and temperature," Appl.Phys. Lett. 94 ((March (13)) (2009)).

17 Wang, P. F., Brambilla, G., Ding, M., Semenova, Y., Wu, Q., Farrell, G. "High-sensitivity, evanescent field refractometric sensor based on a tapered,multimode fiber interference," Opt. Lett. 36, 2233-2235 ((June (12)) (2011)).

18 Ji, W. B., Liu, H.H.,Tjin, S.C., Chow, K.K., Lim, A. "Ultrahigh sensitivity refractive indexsensor based on optical microfiber," IEEE Photon. Technol. Lett. 24, 1872-1874 ((October (20))(2012)).

19 Galina-Nemova, R. K. "Theoretical model of a planar integrated refractive index sensor based on surface plasmon-polariton excitation," Opt. Commun. 275, 76-82 (2007).

20 Zhou, K., et al. "Optic sensors of high refractive-index responsivity and low thermal cross sensitivity that use fiber Bragg gratings of $80^{\circ}$ tilted structures," Optics Letters 31(9), 1193-1195 (2006).

21 Zhang, L., Zhou, K., Chen, X., and Bennion, I. " Low thermal sensitivity grating devices based on ex-45 tilting structure capable of forward-propagating cladding modes coupling," J. Lightw. Technol. 24, 5087-5094 (Dec. 2006). 
22 Margulis, V. A., and Gaiduk, E.A. " Nature of near-infrared absorption in single-wallcarbon nanotubes," Phys. Lett. A 281, 52-58 ((March (1)) (2001)).

23 Set, S. Y., Yaguchi, H., Tanaka, Y., and Jablonski, V. "Laser mode locking using a saturableabsorber incorporating carbon nanotubes," J. Lightw. Technol. 22, 51-56 ((January (1))(2004)).

24 Chow, K. K., Yamashita, S., Set, S.Y. "Four-wave-mixing-based wavelength conver-sion using a single-walled carbon-nanotube-deposited planar lightwave circuit waveguide," Opt. Lett. 35 (12) 2070-2072 ((2010) ).

25 Mihailov, S. L., Walker, R. B., Stocki T. J., and Johnson, D. C. "Fabrication of tilted fibre grating polarisationdependent loss equalizer," Electron. Lett. 37, 284-286 (2001).

26 Giordani, S., Bergin, S. D., Nicolosi V., Lebedkin S., Kappes, M.M. "Debundling of single-walled nanotubes by dilution: observation of large populations of individual nanotubes in amide solvent dispersions," JPhysChemB 110, 15708-15718 (2006).

27 Hasan, T., Scardaci, V. "Stabilization and "debundling" of single-wall carbon nanotube dispersions in Nmethyl-2-pyrrolidone (NMP) by polyvinylpyrrolidone (PVP)", J PhysChem C 111, 12594-12602 (2007).

28 Wang, Y., and Yeow, J. T. "A review of carborn nanotubes-based gas sensors," J. Sensors 2009, 1-24 (May 2009).

29 Stegall, D. B., and Erdogan, T. "Leaky cladding mode propagation in long-period fibergrating devices," IEEE Photon. Technol. Lett. 11, 343-345 ((March (3)) (1999)).

30 Macleod, H. A. [Thin-Film Optical Filters, fourth ed.], CRC Press/Taylor \& Francis,Boca Raton, FL, ch.4, (2010). 